\title{
Analysis of the practical force accuracy of electromagnet-based nanoindenters
}

\author{
Yong Huan ${ }^{\mathrm{a}}$, Dongxu Liu ${ }^{\mathrm{b}}$, Rong Yang ${ }^{\mathrm{a}}$, Taihua Zhang ${ }^{\mathrm{a}, *}$ \\ ${ }^{a}$ State Key Laboratory of Nonlinear Mechanics (LNM), Institute of Mechanics, Chinese Academy of Sciences, Beijing 100190, People's Republic of China \\ ${ }^{\mathrm{b}}$ Department of Civil and Environmental Engineering, University of California, Irvine, CA 92697, USA
}

\section{A R T I C L E I N F O}

\section{Article history:}

Received 21 July 2009

Received in revised form 6 April 2010

Accepted 13 April 2010

Available online 18 April 2010

\section{Keywords:}

Nanoindenter

Force

Accuracy

Measurement

\begin{abstract}
A B S T R A C T
Two important factors that influence the force accuracy of the electromagnet-based nanoindenters but have not yet attracted much attention are analyzed, and a more reasonable way to estimate the force accuracy is presented in this paper. MTS Nano Indenter ${ }^{\circledR}$, with the characteristics of a coil suspended in a uniform magnetic field by two sets of springs acting as an actuator and force measuring unit, is used as an example. One of the two factors is the uniformity of the magnetic field. The other is the stiffness of the supporting spring. Consequently, the practical force accuracy varies considerably from test to test because it firmly depends on the working position of the coil and the displacement stroke. A reasonable estimated accuracy value is of the order of $10^{\circ} \mu \mathrm{N}$ for typical indentation tests with a $10^{2} \mathrm{~nm}$ indentation depth or a $10^{0} \mathrm{mN}$ test force.
\end{abstract}

(c) 2010 Elsevier Ltd. All rights reserved.

\section{Introduction}

The nanoindenter has become a main measurement tool in recent years in the micro/nano-mechanics and materials field owing to its capability for high resolution force measurements [1-4]. There are several types of nanoindenters, for example, MTS Nano Indenter ${ }^{\circledR}$ (incorporated into Agilent in 2008), Hysitron TriboIndenter ${ }^{\circledR}$, CSM Nano Hardness Tester ${ }^{\circledR}$, MML NanoTest ${ }^{\circledR}$ and CSIRO UMIS ${ }^{\circledR}$. Among them, MTS Nano Indenter ${ }^{\circledR}$ is the earliest commercial instrument and now has worldwide popularity. It has a coil-magnet component as the actuator and force measuring unit. In addition, CSM Nano Hardness Tester ${ }^{\circledR}$ and MML NanoTest ${ }^{\circledR}$ have a similar actuator and force measuring unit.

There have been many studies on factors influencing indentation test results, for example, indenter tip shapes, capillary force and the surface roughness of samples [5$8]$, but little investigation has focused on achievable force accuracy. The manufacturers usually like to emphasize the high resolution of the force, which has reached up to

\footnotetext{
* Corresponding author. Tel.: +86 1082543950.

E-mail address: zhangth@Inm.imech.ac.cn (T. Zhang).
}

$50 \mathrm{nN}$ so far. However, the practical force accuracy has never been given in specifications for these instruments. It should be noted that accuracy is a totally different parameter to the resolution. The former is usually defined as being near the true value, and the latter is defined as the fineness of detail that can be distinguished. It is obvious that accuracy can have a major detrimental impact on any final test results. This raises the question of why the very important parameter of force accuracy is invariably absent from nanoindenter specification documents.

One of the major factors is that it is very difficult to calibrate so tiny a force, for example the $50 \mathrm{nN}$ mentioned above. The smallest traceable force currently reported is $5 \mu \mathrm{N}[9,10]$. However, here, the root reason is that the mechanical response of the instrument and the sample are coupled together. The force on the sample (practical force) is not equal to the directly measured magnetic force. However, the calibration process recommended by ISO14577-2:2002 [11] deals only with the magnetic force and not the force on the sample. The key question is what dominates the practical force accuracy of an electromagnet-based nanoindenter. In this study, MTS Nano Indenter ${ }^{\circledR}$ XP used as an example, two important factors influencing the force accuracy are analyzed and a 
more reasonable way to estimate the force accuracy is presented.

\section{Mechanics model of MTS Nano Indenter ${ }^{\circledR}$}

The structure of MTS Nano Indenter ${ }^{\circledR}$ is shown in Fig. 1a, which consists of a coil suspended in a uniform magnetic field by two sets of springs. When a current passes through the coil, an electromagnet force is generated to drive the indenter. If the magnet density $B$ is a constant, the conducted magnetic force $F_{e}(t)$ is proportional to the excitation current $I(t)$, i.e. $F_{e}(t) \propto I(t)$. Thus, the magnetic force can easily be measured by recording the current:

$F_{e}(t)=B l I(t)=D I(t)$

where $l$ is the lead length of the coil, and $D$ is defined as the force sensitivity.

However, the measured magnetic force is still not the force on the sample. To eliminate the possible existing friction, the probe, the coil and the capacitive displacement sensor are all supported and oriented by two sets of springs instead of a conventional bearing assembly. This introduces an extra spring force. In static tests, all damping can be ignored. The mechanics model is shown in Fig. 1b. Here, $M$ is the moving mass, including the coil, probe, displacement sensor and indenter. $K_{f}$ is the stiffness of the machine frame. $K_{s}$ is the stiffness of the spring in the vertical direction. $S$ is the stiffness of the sample. The relationship between the force on the sample $F_{s}(t)$ and the measured magnet force $F_{e}(t)$ is given by:

$F_{s}(t)=F_{e}(t)-K_{s} \times \operatorname{Disp}(t)$

where $\operatorname{Disp}(t)$ is the displacement of the coil, which, in fact, establishes that the force on the sample $F_{s}(t)$ relies on the stiffness $K_{s}$.

Based on the analysis above, the accuracy of the force on the sample $F_{s}(t)$ is influenced by two factors. The first is the uniformity of the magnetic field, which influences the accuracy of the magnetic force $F_{e}(t)$. The second is the stiffness of the spring, which combines with the displacement $\operatorname{Disp}(t)$ to take effect.

\section{Experiments and analysis}

\subsection{Uniformity of magnetic field}

The coil-magnet unit of MTS Nano Indenter ${ }^{\circledR}$ is illustrated in Fig. 2a. A coil is located at the orifice of the fixed magnet. The relationship between the magnet density $B$ and the coil position $Z$ is illustrated in Fig. 2b. As mentioned above, the linear relationship $F_{e}(t) \propto I(t)$ can only be satisfied in the uniform segment of the magnetic field, marked with a dashed box in Fig. $2 b$.

However, in practice, the magnet density $B$ always fluctuates more or less throughout the whole displacement stroke. In other words, the force sensitivity $D$ is not constant all the time, which results in the measurement error of the magnetic force $F_{e}(t)$. This raises the question of how uniform the magnetic field can be kept in practice.

The force sensitivity-position curve of MTS Nano Indenter $^{\circledR}$ XP is shown in Fig. 3. The fluctuation of the force sensitivity along with the position indicates the uniformity of the magnetic field. The mean sensitivity is $89.6 \mathrm{mN} / \mathrm{V}$ throughout the $\pm 0.75 \mathrm{~mm}$ displacement. The maximum fluctuation of the force sensitivity is about $8 \%$. This means the uniformity of the magnetic field is not very good.

Fortunately, the maximum indentation depth of MTS Nano Indenter ${ }^{\circledR} \mathrm{XP}$, described in the manual, is $0.5 \mathrm{~mm}$. Here if the work segment is confined to +0.30 to $+0.75 \mathrm{~mm}$, as marked with a dashed box in Fig. 3, the force sensitivity only changes from $92.6 \mathrm{mN} / \mathrm{V}$ to $93.0 \mathrm{mN} / \mathrm{V}$. In this segment, the mean force sensitivity is $92.8 \mathrm{mN} / \mathrm{V}$ throughout the $0.45 \mathrm{~mm}$ displacement. The maximum fluctuation is about $0.24 \%$. That is, the non-uniformity of the magnetic field can introduce about $0.24 \%$ error to the measured magnetic force $F_{e}(t)$ if the displacement stroke is limited in $0.45 \mathrm{~mm}$.

\subsection{Spring stiffness}

As mentioned above, the force on the sample $F_{s}(t)$ is also influenced by the stiffness of the supporting spring. For MTS Nano Indenter ${ }^{\circledR}$ XP, the force-displacement curve of the supporting spring is shown in Fig. $4 \mathrm{a}$, and the stiff-

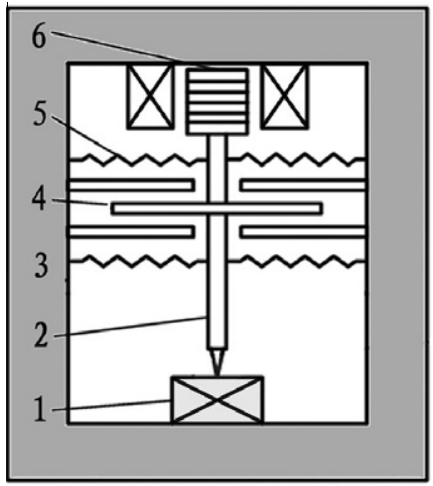

(a)

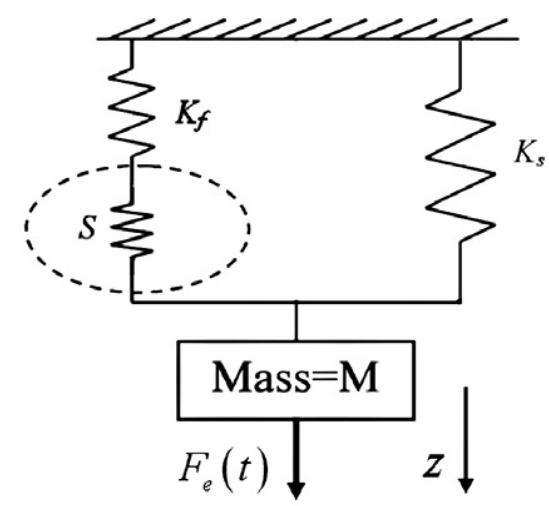

(b)

Fig. 1. MTS Nano Indenter ${ }^{\circledR}$ : (a) structure sketch, (b) mechanics model. 


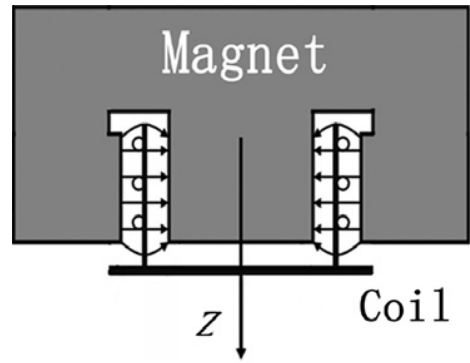

(a)

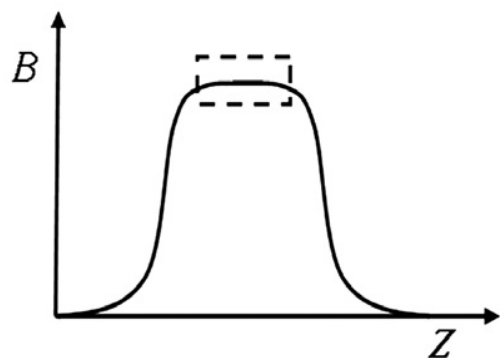

(b)

Fig. 2. Schematic illustration of the coil-magnet component of MTS Nano Indenter ${ }^{\circledR}$ : (a) a coil is located at the orifice of the fixed magnet, (b) the relationship between the magnet density $B$ and the coil position $Z$. MTS Nano Indenter ${ }^{\circledR}$ functions in the uniform segment of the magnetic field (marked with a dashed box).

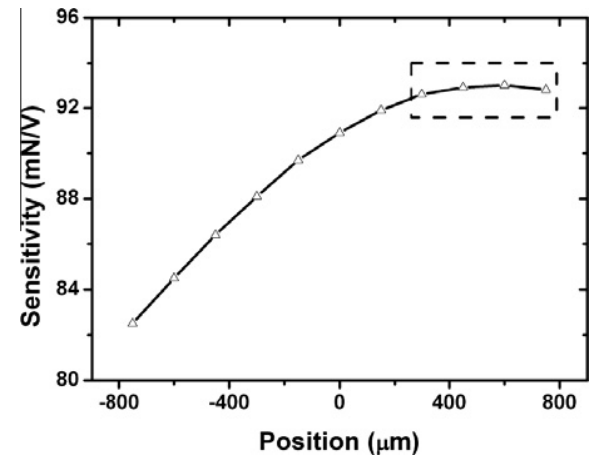

Fig. 3. Relationship between the force sensitivity and the position. The change of the force sensitivity indicates the non-uniformity of the magnetic field.

ness-displacement curve is shown in Fig. 4b. The mean stiffness throughout the $\pm 0.85 \mathrm{~mm}$ stroke is $91.96 \mathrm{~N} / \mathrm{m}$ and the fluctuation is up to $\pm 16 \%$. However, in the Eq. (2), the stiffness is regarded as a constant. Therefore, the fluctuation of the stiffness results in a measurement error of the force on the sample.

The issue is how much error can be introduced in practice by this problem. A typical indentation test result on fused silicon is shown in Fig. 5a. The beeline segment

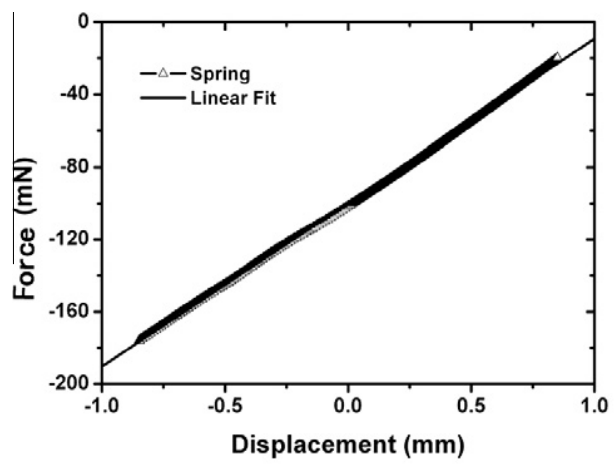

(a) before the zero force point shows the mechanical behavior of the supporting spring. The detail of this segment is shown in Fig. 5b. It is virtually no longer a beeline. The maximum force deviation is about $3 \mu \mathrm{N}$ for a $750 \mathrm{~nm}$ displacement. Unfortunately, we cannot locate this segment with certainty on the whole force-displacement curve of the spring. Therefore, the force deviation for the next $1208 \mathrm{~nm}$ displacement into the surface cannot definitively be established. The estimated value is of the order of $10^{0} \mu \mathrm{N}$, no matter how small the maximum test force is. That is, the force error introduced by the spring is of the order of $10^{\circ} \mu \mathrm{N}$, which is much greater than the force resolution of $50 \mathrm{nN}$ described in the specifications.

\subsection{Analysis}

The first part of the error introduced by the uniformity of the magnetic field is proportional to the measured magnetic force (approximate to the test force). In a typical indentation test with the maximum force of $10^{2} \mathrm{mN}$, this part of the error can be up to $10^{2} \mu \mathrm{N}$. However, this part of the error will always be about $0.24 \%$ of the test force, which is acceptable for the nano/micro-mechanics test.

However, the second part of the error introduced by the spring only depends on the indenter's displacement and has no relationship with the test force. For example, in a microcantilever bending test, the test force is $10 \mu \mathrm{N}$ and the force

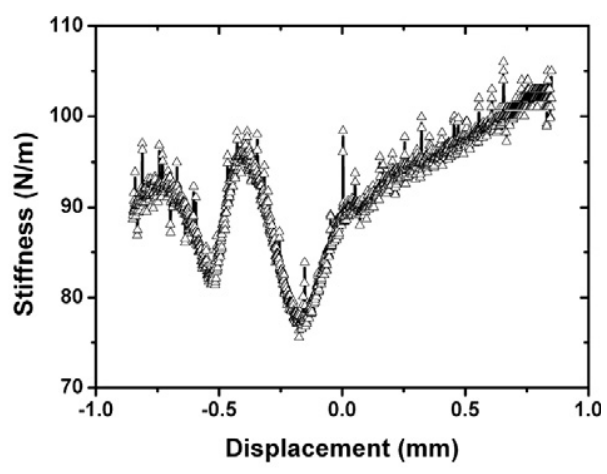

(b)

Fig. 4. Mechanical properties of the supporting spring: (a) force-displacement curve of the supporting spring, (b) stiffness-displacement curve. 


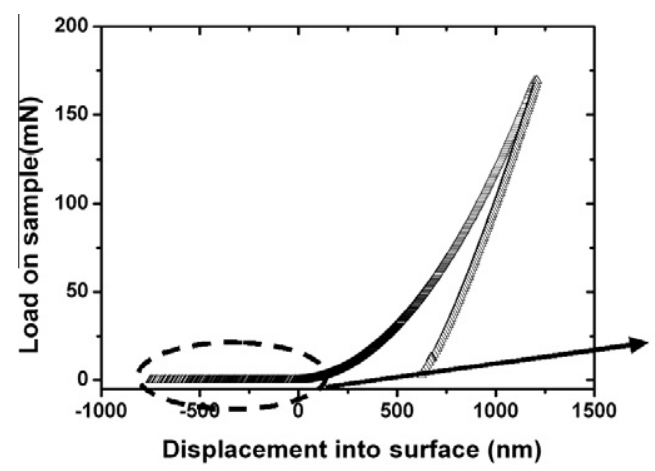

(a)

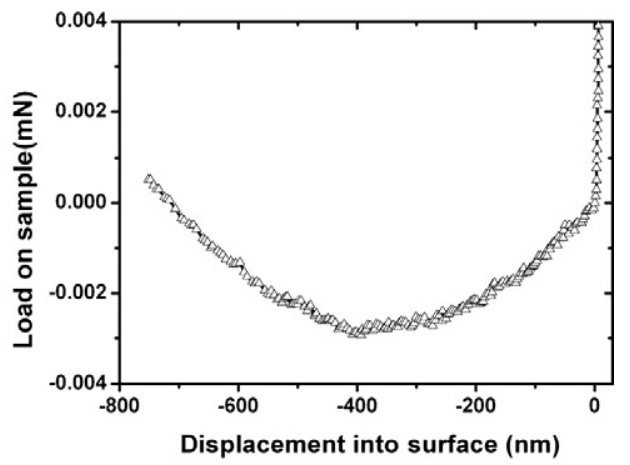

(b)

Fig. 5. (a) A typical indentation test result on fused silicon. (b) The detail of the beeline segment before the force zero point of the indention test.

error introduced by the spring may still be $3 \mu \mathrm{N}$ if the indenter's displacement is still $750 \mathrm{~nm}$. It is obvious that $30 \%$ of the force error is unacceptable in any mechanical tests.

Therefore, the total force error cannot be determined with certainty by simply adding or subtracting the two parts of the error. A reasonable estimated accuracy value is of the order of $10^{\circ} \mu \mathrm{N}$ for typical indentation tests with a $10^{2} \mathrm{~nm}$ indentation depth or a $10^{\circ} \mathrm{mN}$ test force.

\section{Conclusions}

Two important factors influencing the force accuracy of the electromagnet-based nanoindenter are discussed in this article. One is the uniformity of the magnetic field. The other is the stiffness of the supporting spring.

It should be noted that the force accuracy of this kind of nanoindenter firmly depends on the working position of the coil and the displacement stroke, which can vary considerably in different tests. Therefore, the force accuracy cannot be calibrated consistently. This may be the underlying reason why there is no definite specification of the force accuracy in these instruments' manuals.

In addition, an important suggestion for increasing the force accuracy is to improve the linearity of the supporting spring.

\section{Acknowledgments}

This work was supported by the National Natural Science Foundation of China (Grant Nos. 10602058,
10872200) and the LNM initial funding for young investigators.

\section{References}

[1] W.C. Oliver, G.M. Pharr, An improved technique for determining hardness and elastic-modulus using load and displacement sensing indentation experiments, J. Mater. Res. 7 (1992) 1564-1583.

[2] S.H. Hong, K.S. Kim, Y.-M. Kim, et al., Characterization of elastic moduli of $\mathrm{Cu}$ thin films using nanoindentation technique, Compos. Sci. Technol. 65 (2005) 1401-1408.

[3] W.D. Shen, J. Sun, Z.Q. Liu, et al., Methods for studying the mechanical and tribological properties of hard and soft coatings with a nano-indenter, JCT Res. 1 (2) (2004) 117-125.

[4] C.J. Wilson, A. Ormeggi, M. Narbutovskih, Fracture testing of silicon microcantilever beams, J. Appl. Phys. 79 (5) (1996) 2386-2393.

[5] S. Baek, S.K. Cho, C.S. Seok, A correction of geometric error of nanoindenter using atomic force microscope and finite element method, in: Advanced Nondestructive Evaluation I, PTS 1 and 2, Proceedings, vols. 321-323, 2006, pp. 129-131.

[6] S.H. Chen, A.K. Soh, The capillary force in micro- and nanoindentation with different indenter shapes, Int. J. Solids Struct. 45 (10) (2008) 3122-3137.

[7] J. McMinis, R. Crombez, E. Montalvo, Determination of the crosssectional area of the indenter in nano-indentation tests, Physica B Condens. Matter 391 (1) (2007) 118-123.

[8] C.A. Clifford, M.P. Seah, Modelling of nanomechanical nanoindentation measurements using an AFM or nanoindenter for compliant layers on stiffer substrates, Nanotechnology 17 (21) (2006) 5283-5292.

[9] S. Mekid, B. Lim, Design of a mobile indenter for use in the nano range, Nanotechnology 18 (43) (2007) (Art. No. 435501).

[10] J.R. Pratt, J.A. Kramar, D.B. Newell, D.T. Smith, Review of SI traceable force metrology for instrumented indentation and atomic force microscopy, Meas. Sci. Technol. 16 (2005) 2129-2137.

[11] ISO14577-2:2002 Metallic Materials-Instrumented Indentation Test for Hardness and Materials Parameters - Part 2: Verification and Calibration of Testing Machines. 